\title{
Editorial
}

Psychotherapy

and Psychosomatics

\section{The Clinical Inadequacy of Evidence-Based Medicine and the Need for a Conceptual Framework Based on Clinical Judgment}

\author{
Giovanni A. Fava ${ }^{a}$ c Jenny Guidia Chiara Rafanelli ${ }^{a} \quad$ Nicoletta Sonino ${ }^{b, c}$ \\ ${ }^{a}$ Department of Psychology, University of Bologna, Bologna, and ${ }^{\mathrm{b}}$ Department of Statistical Sciences, University of \\ Padova, Padova, Italy; ${ }^{C}$ Department of Psychiatry, State University of New York at Buffalo, Buffalo, N.Y., USA
}

The gap between clinical guidelines developed by evidence-based medicine (EBM) and the real world of clinicians and patients has been widely recognized. There is currently little evidence that EBM has actually improved patient care [1]. It is thus not surprising that most of the time clinicians rely more on their own experience and that of their colleagues (tacit knowledge or 'mindlines') than on explicit evidence from research [2]. Feinstein and Horwitz [3] were among the first to warn about excessive reliance on randomized controlled trials (RCT) and meta-analyses that were not intended to answer questions about the treatment of individual patients. The results of these types of trials may show comparative efficacy of treatments for the average randomized patient but not for those whose characteristics, such as severity of symptoms, comorbidity and other clinical features, depart from standard presentations [3]. In addition, meta-analyses often include highly heterogeneous studies and ascribe conflicting results to random variability, whereas different outcomes may reflect different patient populations, enrollment and protocol characteristics [4]. Even though personalized medicine, described as genomicsbased knowledge, has promised to approach each patient as the biological individual he/she is, the practical applications still have a long way to go and neglect of social and behavioral features may actually lead to 'depersonalized' medicine [5]. Other limitations have emerged over time.
Each therapeutic act may be seen as a result of multiple ingredients that may be specific or nonspecific [6]. Expectations, preferences, motivation, and patient-doctor interactions are examples of variables that may affect treatment outcome [6, 7]. The changed spectrum of medical disorders (shifted toward aging and chronicity) and the interindividual differences in health priorities make the focus on single diseases potentially misleading, whereas there is growing awareness that the aim of the treatment should refer to personal goals (from attainment of cure to prevention of recurrence, from removal of functional impairment to alleviation of symptoms) [8]. EBM does not do justice to the importance of these interactions and provides an oversimplified and reductionistic view of treatment. Finally, the presence of investigators with substantial financial conflicts of interest in panels concerned with clinical guidelines and the excessive reliance of metaanalyses on industry-funded studies constitute two major sources of bias in literature interpretation $[1,9]$.

Horwitz et al. [10] developed a method of clinical inquiry within RCT that can enhance the applicability of results to clinical decision making. Reanalyzing the BetaBlocker Heart Attack Trial, they found that propranolol reduced the risk of dying for the 'average' patient who survived an acute myocardial infarction, whereas it was harmful in a subgroup characterized by specific cotherapy histories. If we accept the possibility that a treatment

\section{KARGER 125}

(c) 2014 S. Karger AG, Base

0033-3190/14/0841-0001\$39.50/0

E-Mail karger@karger.com

www.karger.com/pps
Giovanni A. Fava, MD

Department of Psychology, University of Bologna

Viale Berti Pichat 5

IT-40127 Bologna (Italy)

E-Mail giovanniandrea.fava@unibo.it 
which is helpful on average may be ineffective in some and even harmful in others, we may learn that a given therapy may not be of value for a particular class or subgroup of subjects who are defined in terms of more detailed (compared to the RCT eligibility criteria) specifications of clinical conditions [10]. Recently, Richardson and Doster [11] have suggested the consideration of the following three dimensions in the process of evidencebased decision making: baseline risk of poor outcomes from an index disorder without treatment, responsiveness to the treatment option and vulnerability to the adverse effects of treatment. EBM is focused on the potential benefits that therapy may entail as to baseline risk, but it is likely to neglect the other two dimensions. A rational approach to treatment takes into account the balance between potential benefits and adverse effects applied to the individual patient [12]. The achievement of such a balance is hindered by the difficult integration of different sources of information. Guidelines tend to place emphasis on systematic reviews and meta-analyses of RCT that are uniquely geared to highlighting benefits [12]. The appraisal of adverse effects relies primarily on observational studies and data from routine clinical practice and may not emerge from RCT, unless these effects occur early in the course of treatment and are specifically investigated [12].

The clinician needs to have a clear account of the potential benefits of a specific treatment as well as of the predictors of responsiveness and of the potential adverse events that may be triggered by the therapeutic act. For instance, antidepressant drugs have been found to be effective in the treatment of major depressive disorders, but the magnitude of benefit of antidepressant medication compared with placebo increases with the severity of depression [13]. If a patient suffers from severe depression there is little doubt that pharmacotherapy may yield benefits, even though response may vary from patient to patient [14]. If symptoms are of mild or moderate intensity, the benefits may be minimal or nonexistent, while adverse effects may be substantial, such as switching from a unipolar to a bipolar course. Unfortunately, the prescribing clinician is driven by an overestimated consideration of potential benefits, paying little attention to the likelihood of responsiveness and to potential vulnerabilities in relation to the adverse effects of treatment [14].

The conceptual model that has generated EBM and guidelines clashes with clinical reality and fosters a dichotomy between medical science and clinical judgment. EBM has certainly made an important contribution to questioning unsubstantiated therapeutic claims. The time has come, however, to become more aware of its considerable limitations, including overall reductionism, disregard of patient-physician relationships and patient preferences and insufficient consideration of problems related to financial conflicts of interest. As an increasing body of literature indicates [1], EBM offers only a restrictive interpretation of the scientific approach to clinical practice.

Endorsement of a more comprehensive conceptual framework, including the issues of responsiveness and vulnerability (in addition to baseline risk) and the consideration of comorbidity and multimorbidity, as well as other important contextual factors [11], may lead to more effective decision making. This alternative conceptual model is centered primarily on clinical judgment. In 1967, Feinstein [15] urged clinicians to develop a 'basic science' of their own, to study the clinical phenomena directly, to assess the importance of different types of clinical data, to create appropriate systems of taxonomy for classifying the information, and to develop intellectual models and pragmatic methods that would enlighten the clinical process. Such a line of research $[16,17]$ would call for a different conception of clinical trials, where the aim is not the simple demonstration of statistical superiority of a treatment compared to controls in a short-term study, but also the appraisal of differential effects according to clinical characteristics and of adverse events that persist over time in a long-term perspective. These latter events have been recently defined as iatrogenic comorbidity [7]. The data that may originate from this type of clinical trial are closer to offer what a clinician needs for assessing treatment options in the individual case. Such trials are unlikely to be supported by the private industry and call for a renewed interest by public funding agencies. The model is realistic instead of idealistic, and in its comprehensive consideration of complex variables may give a new input to clinical research. It may be frustrating to those who have little familiarity with the clinical process, but it is more in keeping with what the physician faces in everyday patient management.

\section{Acknowledgment}

The work of Dr. Guidi and Dr. Rafanelli was supported in part by a grant from Compagnia di San Paolo, Torino, Italy.

\section{Disclosure Statement}

The authors have no conflicts of interest to disclose. 


\section{References}

$>1$ Every-Palmer S, Howick J: How evidencebased medicine is failing due to biased trials and selective publication. J Eval Clin Practice 2014;doi:10.11111/jep.12147.

$>2$ Gabbay J, le May A: Evidence-based guidelines or collectively constructed 'mindlines'? Ethnographic study of knowledge management in primary care. BMJ 2004;329:1013.

$\checkmark 3$ Feinstein AR, Horwitz RI: Problems in the 'evidence' of 'evidence-based medicine'. Am J Med 1997;103:529-535.

$>4$ Jane-Wit D, Horwitz RI, Concato J: Variation in results from randomized, controlled trials: stochastic or systematic? J Clin Epidemiology 2010;63:56-63.

$>5$ Horwitz RI, Cullen MR, Abell J, Christian JB: (De)personalized medicine. Science 2013; 339:1155-1156.

6 Rickels K (ed): Non-Specific Factors in Drug Therapy. Springfield, Thomas, 1968.
7 Fava GA, Tomba E, Tossani E: Innovative trends in the design of therapeutic trials in psychopharmacology and psychotherapy. Prog Neuropsychopharmacol Biol Psychiatry 2013;40:306-311.

8 Reuben DB, Tinetti ME: Goal-oriented patient care - an alternative health outcomes paradigm. N Engl J Med 2012;366:777-779.

-9 Fava GA: Meta-analyses and conflict of interest. CNS Drugs 2012;26:93-96.

$>10$ Horwitz RI, Singer BH, Makuch RW, Viscoli CM: Can treatment that is helpful on average be harmful to some patients? J Clin Epidemiol 1996;49:395-400.

11 Richardson WS, Doster LM: Comorbidity and multimorbidity need to be placed in the context of a framework of risk, responsiveness, and vulnerability. J Clin Epidemiol 2014; 67:244-246.
12 Vanderbroucke JP, Psaty BM: Benefits and risks of drug treatments. How to combine the best evidence on benefits with the best data about adverse effects. JAMA 2008;300:24172419.

13 Fournier JC, DeRubeis RJ, Hollon SD, Dimidjian S, Amsterdam JD, Shelton RC, Fawcett $\mathrm{J}$ : Antidepressant drug effects and depression severity. JAMA 2010;303:47-53.

14 Fava GA. Rational use of antidepressant drugs. Psychother Psychosom 2014;83:197204

15 Feinstein AR: Clinical Judgment. Baltimore, Williams \& Wilkins, 1967.

16 Weiss P, Guidi J, Fava M: Closing the efficacyeffectiveness gap. J Clin Psychiatry 2009;70: 446-449.

17 Fava GA, Rafanelli C, Tomba E: The clinical process in psychiatry. J Clin Psychiatry 2012; 73:177-184. 